\title{
Pulmonary Embolism in Pneumonia: Still a Diagnostic Challenge? Results of a Case-Control Study in 100 Patients
}

\author{
Maria Paparoupa, ${ }^{1}$ Loukia Spineli, ${ }^{2}$ Theodor Framke, ${ }^{2}$ Huy Ho, \\ Frank Schuppert, ${ }^{3}$ and Adrian Gillissen ${ }^{4}$ \\ ${ }^{1}$ Intensive Care Unit, University Hospital of Giessen, 35392 Giessen, Germany \\ ${ }^{2}$ Institute of Biostatistics, Hannover Medical School (MHH), 30625 Hannover, Germany \\ ${ }^{3}$ Department of Gastroenterology, Endocrinology, Diabetology and General Medicine, Klinikum Kassel, 34125 Kassel, Germany \\ ${ }^{4}$ Department of Pulmonary Medicine, Klinikum Kassel, 34125 Kassel, Germany
}

Correspondence should be addressed to Maria Paparoupa; maria.paparoupa@yahoo.com

Received 12 February 2016; Revised 20 April 2016; Accepted 8 May 2016

Academic Editor: Dennis W. T. Nilsen

Copyright (C) 2016 Maria Paparoupa et al. This is an open access article distributed under the Creative Commons Attribution License, which permits unrestricted use, distribution, and reproduction in any medium, provided the original work is properly cited.

\begin{abstract}
This study evaluated the diagnostic value of D-dimer, CRP, and leucocytes count to detect an underlying pulmonary embolism (PE) in patients with pneumonia. A predictive model of an underlying PE, based on laboratory markers and clinical symptoms, was our ultimate objective. Overall 100 patients underwent a computed tomography angiography (CTA) of the lung: 54 with coexistence of $\mathrm{PE}$ and pneumonia (cases) and 46 with pneumonia without PE (controls). Cases and controls were matched 1:1. Symptoms and paraclinical findings were registered on admission. Receiver operating characteristic (ROC) curves, search for an optimal threshold, and conditional logistic regression analysis were conducted. D-dimer has a moderate ability to detect PE in pneumonia. Sensitivity of D-dimer was estimated at $97.78 \%$ and specificity at $11.11 \%$. No optimal cut-point has acceptable diagnostic ability. After excluding patients with sepsis, sensitivity was reduced to $96.97 \%$, whereas specificity increased to $16.13 \%$. Consolidation in chest X-ray and positive D-dimer predict better an underlying PE as D-dimer itself. Thus, discriminatory power of the prediction model (AUC of 0.740 ) is not much greater than D-dimer (AUC of 0.703 ). No threshold that could increase the diagnostic value of $\mathrm{D}$-dimer or a prediction model which is significantly better than D-dimer itself was identified.
\end{abstract}

\section{Introduction}

The coexistence of pulmonary embolism (PE) and pneumonia (known as infarction pneumonia) is a common diagnosis in the setting of emergency medicine worldwide. The differential diagnosis towards community acquired pneumonia (CAP) remains difficult and requires the performance of a computed tomography pulmonary angiography (CTPA). The term "pneumonia" is used to describe both subtypes of pneumonia that are compared in this paper: pneumonia after pulmonary embolism which is mentioned as "infarction pneumonia" and pneumonia caused by bacterial or viral pathogens without pulmonary infarction, which is mentioned as "community acquired pneumonia."

The existence of an underlying PE when pneumonia is diagnosed is of a highly therapeutic importance, as in this case initiation of anticoagulation treatment is required. Moreover, the computed angiography of the lung is an expensive and possibly harmful method, due to radiation and iodine exposure, which may lead to serious adverse events. Therefore, a prognostic model of an underlying PE, based only on patient's symptoms and paraclinical findings on admission, could be extremely useful in order to select cases with a high PE possibility which should undergo CTPA, in order to confirm or exclude the diagnosis. This clinical practice could contribute to the reduction of underdiagnosed cases. On the other hand, unnecessary performance of a computed tomography can be avoided in patients with low pulmonary embolism profile.

$\mathrm{D}$-dimer is a laboratory biomarker that may be a candidate diagnostic test for the diagnosis of $\mathrm{PE}$, since it has been used for the detection of thrombus. The cut-offs for D-dimer 
concentration are well known for an underlying PE without pneumonia [1]. However, the cut-offs for this parameter are unknown when pulmonary embolism coexists with pneumonia. It should be noted that D-dimer concentration increases considerably in patients with sepsis and, hence, its ability to successfully detect PE is getting dubious.

\section{Objectives of the Study}

The aim of this biomarker Phase II study is to perform a series of evaluations regarding the diagnostic value of easily and fast provided laboratory parameters such as D-dimers, CRP, and leucocytes count to successfully detect an underlying PE in patients with pneumonia. In addition, the diagnostic value of D-dimer as a marker of an underlying PE will be reevaluated after excluding patients with sepsis (sensitivity analysis). Clinical symptoms, such as fever, chest pain, syncope, dyspnea, hemoptysis, and cough, as well as echocardiographic and common X-ray findings will be also assessed between patients with $\mathrm{PE}$ and infarction pneumonia and patients with CAP. A predictive model of an underlying $\mathrm{PE}$ in patients with pneumonia is the ultimate objective of this study.

\section{Study Design and Patients}

In order to predict an underlying $\mathrm{PE}$ in patients with pneumonia, we designed a retrospective case-control study between subjects with coexistence of $\mathrm{PE}$ and infarction pneumonia and subjects with community acquired pneumonia (CAP) without pulmonary infarction. We examined the initial sample size of 600 medical records of patients diagnosed with pneumonia between 2012 and 2014 in the general hospital of Klinikum Kassel, Germany. The diagnosis of pneumonia was made according to radiological criteria, after chest X-ray, computed angiography of the lung, or both. Only 100 individuals had undergone a computed tomography angiography (CTA) of the lung; 54 were diagnosed with coexistence of $\mathrm{PE}$ and infarction pneumonia and 46 were diagnosed with pneumonia without PE (CAP). We selected exclusively these patients for the next step because an underlying PE can only be confirmed or excluded after a CTPA. Subsequently, patients with coexistence of PE and infarction pneumonia (cases) were matched at $1: 1$ ratio with CAP (controls), according to gender and age, in order to prevent confounding issues. Matching was not achieved for 9 cases and 1 control, leading to their exclusion from the data analysis. None of the selected patients had a former history of pulmonary embolism or an oral vitamin-K antagonist medication for any indication.

For each patient major and minor symptoms on admission were registered: fever and chest pain were referred to as major symptoms, whereas dyspnea, cough, dyspnea and cough, hemoptysis, and syncope were referred to as minor symptoms. The categorisation of patients according to their gender, age, and admission symptoms is demonstrated in Table 1.

Laboratory parameters such as C-reactive protein (CRP), total leucocytes count, and D-dimers, as well as the existence of consolidation in chest X-ray, were identified on admission. The D-Dimer Assay used for laboratory testing was "D-Dimere Test Innovance" from Siemens. Only 6 cases underwent directly a CTPA without a conventional chest Xray first (because of the high clinical suspicion of PE). 28 cases fulfilled the criteria of sepsis (SIRS-Systemic Inflammatory Distress Syndrome-and at least one organ failure). Finally, the extension of the PE (central or subsegmental) and echocardiographic findings of a right ventricular hypertension $(\mathrm{RVH})$ were collected only in PE cases. Comorbidities classically combined with RVH were registered using the medical history of each patient (see Tables 2 and 3 ).

\section{Statistical Analysis}

Baseline characteristics of the matched cases-controls were analyzed descriptively and compared with paired $t$-tests for quantitative data whereas McNemar tests and Cohen's Kappa were used for binary and categorical data, respectively, to assess the agreement between the two groups.

4.1. Evaluation of the Diagnostic Value. Receiver operating characteristic (ROC) curves were used for the evaluation of the diagnostic value of the laboratory parameters D-dimer, CRP, and leucocytes count. To interpret the area under the ROC curves (AUC), the traditional academic point system was used; 0.50-0.60 denotes very low diagnostic performance of the test, $0.60-0.70$ low, $0.70-0.80$ moderate, $0.80-0.90$ good, and 0.90-1 excellent performance [2]. The laboratory parameter with at least a moderate ability to discriminate pneumonic patients with underlying PE was selected for the subsequent analysis.

To assess the diagnostic accuracy of the selected laboratory parameters sensitivity and specificity, as well as positive predictive value (PPV) and negative predictive value (NPV), were calculated using a $2 \times 2$ contingency table at a cutpoint of 0.55 . Accuracy was also calculated, as it reflects the probability of a correct test result and the odds ratio (OR) as another useful measure of diagnostic accuracy. Note that all these aforementioned measures were recalculated after excluding patients with sepsis.

4.2. Graphical Analysis for the Optimal Threshold. Optimal thresholds were detected visually using a plot proposed by Harris [3] that illustrates both sensitivity and specificity curves for all possible cut-offs of the selected laboratory parameter. In particular, the coordinates of the intersection of sensitivity with specificity reflect the probability value of sensitivity-specificity equality and the candidate optimal threshold of the studied test.

4.3. Conditional Logistic Regression Analysis for the Prediction Model. Among laboratory parameters evaluated, only those with at least moderate ability to discriminate pneumonic patients with underlying $\mathrm{PE}$ were considered in the prediction model. Clinical symptoms were evaluated regarding their association with the coexistent PE status. In the light of matched cases-controls, the appropriate analysis 
TABLE 1: Categorisation of patients according to their gender, age, and admission symptoms.

\begin{tabular}{|c|c|c|c|}
\hline \multirow{2}{*}{ Characteristic } & \multicolumn{2}{|c|}{ Patient group } & \multirow{2}{*}{ Total $(n=90)$} \\
\hline & $\mathrm{PE}(n=45)$ & $\mathrm{CAP}(n=45)$ & \\
\hline \multicolumn{4}{|l|}{ Gender } \\
\hline Male & $26(58 \%)$ & $26(58 \%)$ & $52(58 \%)$ \\
\hline Female & $19(42 \%)$ & $19(42 \%)$ & $38(42 \%)$ \\
\hline$p$ value (McNemar) & \multicolumn{2}{|c|}{$\mathrm{NA}^{1}$} & \\
\hline \multicolumn{4}{|l|}{ Age } \\
\hline Mean & 66.58 & 66.22 & 66.40 \\
\hline Std & 17.59 & 17.41 & 17.40 \\
\hline Median & 71 & 71 & 71 \\
\hline Minimum & 23 & 25 & 23 \\
\hline Maximum & 92 & 99 & 99 \\
\hline$p$ value $(t$-test $)$ & \multicolumn{2}{|c|}{0.381} & \\
\hline \multicolumn{4}{|l|}{ Fever } \\
\hline Yes & $13(28.89 \%)$ & $12(26.67 \%)$ & $25(27.78 \%)$ \\
\hline No & $32(71.11 \%)$ & $33(73.33 \%)$ & $65(72.22 \%)$ \\
\hline$p$-value (McNemar) & \multicolumn{2}{|c|}{0.808} & \\
\hline \multicolumn{4}{|l|}{ Chest pain } \\
\hline Yes & $24(53.33 \%)$ & $16(35.56 \%)$ & $40(44.44 \%)$ \\
\hline No & $21(46.67 \%)$ & $29(64.44 \%)$ & $50(55.56 \%)$ \\
\hline$p$-value (McNemar) & \multicolumn{2}{|c|}{0.032} & \\
\hline \multicolumn{4}{|l|}{ Other symptoms } \\
\hline Dyspnea & $18(40.00 \%)$ & $16(35.56 \%)$ & $34(37.78 \%)$ \\
\hline Dyspnea and cough & $5(11.11 \%)$ & $15(33.33 \%)$ & $20(22.22 \%)$ \\
\hline Hemoptysis & $0(0.00 \%)$ & $3(6.67 \%)$ & $3(3.33 \%)$ \\
\hline Syncope & $6(13.33 \%)$ & $3(6.67 \%)$ & $9(10.00 \%)$ \\
\hline No other symptoms & $16(35.56 \%)$ & $8(17.78 \%)$ & $24(26.67 \%)$ \\
\hline$p$ value (Kappa) & \multicolumn{2}{|c|}{0.588} & \\
\hline
\end{tabular}

PE: pulmonary embolism; CAP: community acquired pneumonia; $n$ : number of patients; Std: standard deviation; NA: not applicable; $t$-test: paired $t$-test; McNemar: McNemar test; Kappa: weighted Kappa coefficient.

${ }^{1}$ There are no discordant pairs.

is conditional logistic regression that provides conditional estimates of the OR [4]. Specifically, multiple conditional logistic regression models were implemented with dependent variable the probability of "coexistent PE and pneumonia" and independent variables of at least one clinical symptom and the selected laboratory parameter, which was maintained in all studied models. The selection of the best fitted model was based on Akaike's (AIC) criterion; the smaller the AIC value is the better the model fits the data. Then we retrieved the prediction probabilities of the model and used them as the "new diagnostic test." Ultimately, we compared the ROC of the prediction model with the ROC of D-dimer itself.

4.4. Secondary Analysis. As a secondary analysis we assessed the impact of embolism extension and the development of $\mathrm{RVH}$ on D-dimer concentration. A multivariable regression model was employed where D-dimer concentration was the dependent variable, whereas the extension of embolism, the development of $\mathrm{RVH}$, and their interaction were the independent variables.

In all model-based analysis significance was inferred at a level of 0.05 . Please note that significance should not be interpreted in a strict confirmatory sense. All statistical analyses were conducted using SAS 9.3.

\section{Results}

5.1. Demographic and Baseline Characteristics. Matching was successful for 45 pairs of patients with age difference of 2 years on average and a range from 0 up to 7 years. As shown in Table 1, gender was similarly distributed in both groups. Patients were on average age of 66 years old and the majority was male (around 60\%). Fever status was similarly distributed in both groups and was present in approximately one-third of the study population. Chest pain was present mostly in patients with PE and pneumonia (53\%) and it was absent in $64 \%$ of controls. Regarding the category "other symptoms," dyspnea, syncope, and no other symptoms were more present in the PE group, whereas hemoptysis and combination of dyspnea and cough was predominant in the CAP group.

More than a half had an X-ray consolidation in both study groups. However, information on consolidation status could not be retrieved in 6 cases, as these ones underwent directly a CTPA. Approximately one-third of the study subjects in 
TABLE 2: Categorisation of patients according to paraclinical characteristics.

\begin{tabular}{|c|c|c|c|}
\hline \multirow{2}{*}{ Characteristic } & \multicolumn{2}{|c|}{ Patient group } & \multirow{2}{*}{ Total $(n=90)$} \\
\hline & $\mathrm{PE}(n=45)$ & $\mathrm{CAP}(n=45)$ & \\
\hline \multicolumn{4}{|l|}{ Consolidation } \\
\hline Yes & $21(53.85 \%)$ & $30(66.67 \%)$ & $51(60.71 \%)$ \\
\hline No & $18(46.15 \%)$ & $15(33.33 \%)$ & 33 (39.29\%) \\
\hline Missing & 6 & 0 & 6 \\
\hline$p$ value (McNemar) & \multicolumn{2}{|c|}{0.371} & \\
\hline \multicolumn{4}{|l|}{ Sepsis } \\
\hline Yes & $12(26.67 \%)$ & $14(31.11 \%)$ & $26(28.89 \%)$ \\
\hline No & $33(73.33 \%)$ & $31(68.89 \%)$ & $64(71.11 \%)$ \\
\hline$p$ value (McNemar) & \multicolumn{2}{|c|}{0.564} & \\
\hline \multicolumn{4}{|l|}{ D-dimer } \\
\hline Mean & 7.20 & 3.16 & 5.18 \\
\hline Std & 8.27 & 4.67 & 6.98 \\
\hline Median & 4.10 & 1.85 & 2.77 \\
\hline Minimum & 0.52 & 0.18 & 0.18 \\
\hline Maximum & 36.10 & 27.70 & 36.10 \\
\hline$p$ value ( $t$-test) & \multicolumn{2}{|c|}{0.003} & \\
\hline \multicolumn{4}{|l|}{$C R P$} \\
\hline Mean & 98.13 & 107.78 & 102.95 \\
\hline Std & 91.10 & 118.65 & 105.29 \\
\hline Median & 64 & 64 & 64 \\
\hline Minimum & 5 & 3 & 3 \\
\hline Maximum & 404 & 399 & 404 \\
\hline$p$ value $(t$-test $)$ & \multicolumn{2}{|c|}{0.695} & \\
\hline \multicolumn{4}{|l|}{ Leucocytes } \\
\hline Mean & 11671.11 & 12213.33 & 11942.22 \\
\hline Std & 4425.83 & 6343.34 & 5445.29 \\
\hline Median & 10800 & 11600 & 10950 \\
\hline Minimum & 4100 & 4900 & 4100 \\
\hline Maximum & 24400 & 42700 & 42700 \\
\hline$p$ value ( $t$-test) & \multicolumn{2}{|c|}{0.650} & \\
\hline
\end{tabular}

PE: pulmonary embolism; CAP: community acquired pneumonia; $n$ : number of patients; Std: standard deviation; CPR: C-reactive protein; $t$-test: paired $t$-test; McNemar: McNemar test.

TABle 3: Extension of PE and RVH in PE group. Comorbidities in RVH group.

\begin{tabular}{lcc}
\hline Characteristic & Counts & Percentage (\%) \\
\hline Embolism & 21 & \\
Central & 24 & 46.67 \\
Subsegmental & & 53.33 \\
\hline$R V H$ & 18 & \\
Yes & 27 & 40.00 \\
No & & 60.00 \\
\hline Comorbidities $R V H$ & 4 & \\
COPD & 6 & 22.22 \\
LVI & 8 & 33.33 \\
RVH PE & & 44.44 \\
\hline
\end{tabular}

PE: pulmonary embolism; RVH: right ventricular hypertension; COPD: chronic obstructive pulmonary disease; LVI: left ventricular insufficiency.

both groups fulfilled the sepsis criteria (SIRS and at least one organ failure). The average $\mathrm{D}$-dimer value was at least 2 times higher in patients with coexistent PE and pneumonia than in controls and this difference was significant based on paired $t$-test (mean difference 4.04, $p$ value 0.003). Furthermore, patients with coexistent $\mathrm{PE}$ and infarction pneumonia had on average lower CRP values and leucocyte counts than controls, but these differences were not statistically significant ( $p$ value 0.695 and 0.650 , resp.). Among patients with $\mathrm{PE}$, approximately $47 \%$ had a central embolism and $60 \%$ had no echocardiographic signs of RVH. Most interestingly, the majority (55.55\%) of patients with right ventricular hypertension (RVH) had relative comorbidities; $33.33 \%$ left ventricular insufficiency (LVI); and $22.22 \%$ chronic obstructive pulmonary disease (Table 3).

5.2. Evaluating the Diagnostic Value of D-Dimer, CRP, and Leucocytes. To evaluate the diagnostic value of the laboratory parameters D-dimer, CRP, and leucocytes to successfully detect an underlying $\mathrm{PE}$, we compared the receiver operating characteristic curves (ROC) of these parameters. Figure 1 
TABLE 4: Evaluating the diagnostic value of D-dimer using a known threshold.

(a)

\begin{tabular}{lcc}
\hline \multirow{2}{*}{ D-dimer results on PE } & \multicolumn{2}{c}{ CTA results on PE } \\
& Presence & Absence \\
\hline Positive & $44(\mathrm{TP})$ & $40(\mathrm{FP})$ \\
Negative & $1(\mathrm{FN})$ & $5(\mathrm{TN})$ \\
\hline
\end{tabular}

(b)

\begin{tabular}{lccc}
\hline Measure & Proportion & Asymptotic 95\% CI & Exact 95\% CI \\
\hline Sensitivity & $97.78 \%$ & $93.47 \%-100 \%$ & $88.23 \%-99.94 \%$ \\
Specificity & $11.11 \%$ & $1.93 \%-20.29 \%$ & $3.71 \%-24.05 \%$ \\
PPV & $52.38 \%$ & $41.70 \%-63.06 \%$ & $41.19 \%-63.40 \%$ \\
NPV & $83.33 \%$ & $53.51 \%-100 \%$ & $35.88 \%-99.58 \%$ \\
Accuracy & $54.44 \%$ & $44.16 \%-64.73 \%$ & $43.60 \%-64.98 \%$ \\
\hline
\end{tabular}

PE: pulmonary embolism; CTA: computed tomography angiography; TP: true positive; FP: false positive; FN: false negative; TN: true negative, $\mathrm{CI}$ : confidence interval; PPV: positive predictive value; NPV: negative predictive value.

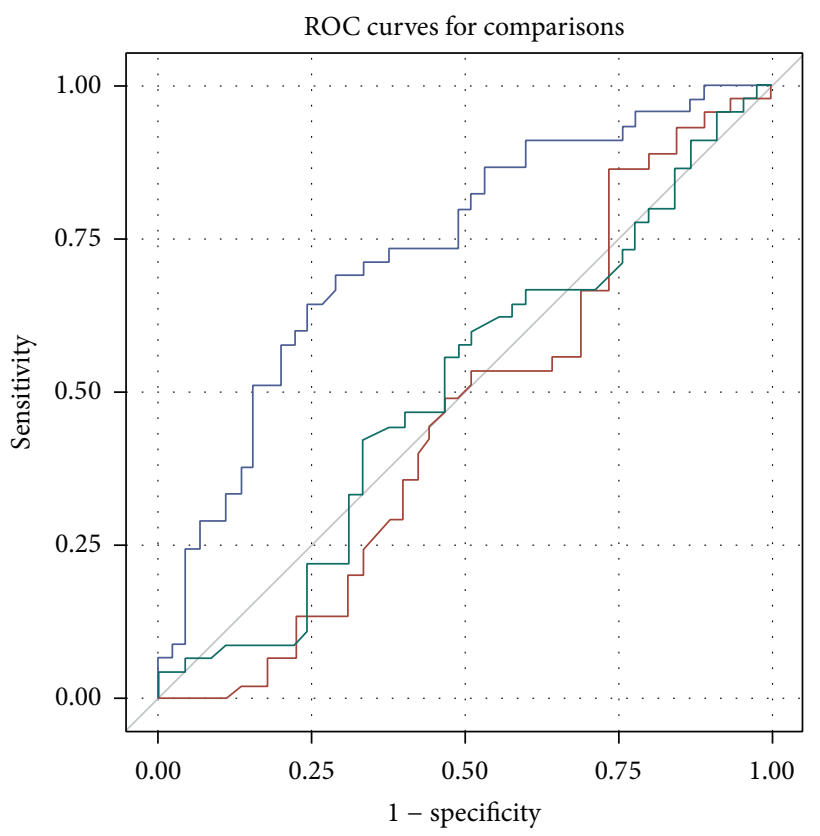

ROC curve (area)

— D-dimer (0.7331)

- Leucocytes (0.5017)

C CRP $(0.4598)$

FIGURE 1: ROC curves for D-dimer, CRP, and Leucocytes.

presents the ROC for each parameter using different colors along with the respective area under the curve (AUC). Ddimer has AUC of 0.733 and, hence, its discriminatory power seems to be moderate. CRP and leucocytes yield an AUC of 0.460 and 0.502 , respectively; both parameters have a very poor diagnostic accuracy and are likely to diagnose patients with coexistent $\mathrm{PE}$ at random.

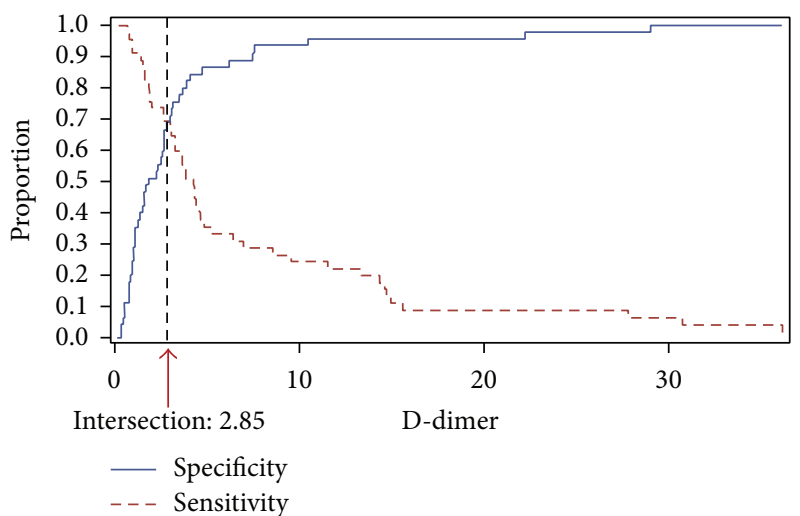

FIgURE 2: Plot by Harris for sensitivity and specificity of D-dimer across all cut-off values.

5.3. Evaluating the Diagnostic Value of D-Dimer Using a Known Threshold. As shown in Tables 4(a) and 4(b), Ddimer achieves high true positive and false positive results as opposed to true negative and false negative results which are very low. Sensitivity is estimated at $97.78 \%$ and specificity at $11.11 \%$ and, therefore, D-dimer is very sensitive for the detection of an underlying PE in the setting of pneumonia, without being specific. PPV indicates that $52 \%$ with a positive test result actually have a PE, whereas the remaining high proportion of $48 \%$ estimated to have PE does not. Furthermore, according to NPV, $83 \%$ of patients with negative test result do not have coexistent $\mathrm{PE}$ and, consequently, the risk of overlooking a PE is actually low. The accuracy of D-dimer was $54.44 \%$.

5.4. Searching for the Optimal D-Dimer Threshold. Using the plot proposed by Harris, sensitivity and specificity intersect at the value of $2.85 \mathrm{mg} / \mathrm{L} \mathrm{FEU}$ which corresponds to sensitivity and specificity equal to $68.89 \%$ (Figure 2). Compared to the cut-off at $0.55 \mathrm{mg} / \mathrm{LFEU}$ used in the study, sensitivity decreased by $36 \%$, whereas specificity increased by $520 \%$. Consequently, D-dimer impairs its ability to correctly detect patients with PE but gained its ability to correctly detect patients without PE. Sensitivity decreases whereas specificity increases with a cut-off value above $2.85 \mathrm{mg} / \mathrm{L}$ FEU. In contrast, sensitivity increases whereas specificity decreases for cut-off values below $2.85 \mathrm{mg} / \mathrm{L}$ FEU. To achieve a sensitivity of at least $90 \%$, the best cut-point could be $0.95 \mathrm{mg} / \mathrm{L} \mathrm{FEU}$, with sensitivity $91.1 \%$ and specificity $24.4 \%$. Using the unweighted Youden index, the optimal cut-point is $0.4 \mathrm{mg} / \mathrm{L} \mathrm{FEU} \mathrm{with}$ corresponding sensitivity of $100 \%$ and specificity of $6.7 \%$. Taking into account these results we conclude that no optimal cut-point for D-dimer seems to reach acceptable diagnostic ability. Excluding the patients with sepsis reduces the sample to 64 patients. Sensitivity is reduced negligibly to $96.97 \%$, whereas specificity increased to $16.13 \%$, which still renders as low D-dimer's specificity ability.

5.5. Prediction Model of an Underlying PE in Patients with Pneumonia. The regression coefficients for the covariates Ddimer and symptoms were interpreted in the exponential 
TABLE 5: Studied covariates along with their levels and the coded values used in the models.

\begin{tabular}{lcc}
\hline Covariate & Levels & Coded value $^{1}$ \\
\hline \multirow{2}{*}{ Chest pain } & No & 0 \\
& Yes & 1 \\
\hline \multirow{2}{*}{ Consolidation } & No & 0 \\
& Yes & 1 \\
\hline \multirow{2}{*}{ Fever } & No & 0 \\
& Yes & 1 \\
\hline \multirow{3}{*}{ Other symptoms } & No other symptoms & 0 \\
& Dyspnea & 1 \\
& Dyspnea and cough & 2 \\
& Hemoptysis & 3 \\
\hline
\end{tabular}

${ }^{1}$ The levels coded with zero are the reference levels to be compared with the rest of the levels in the model.

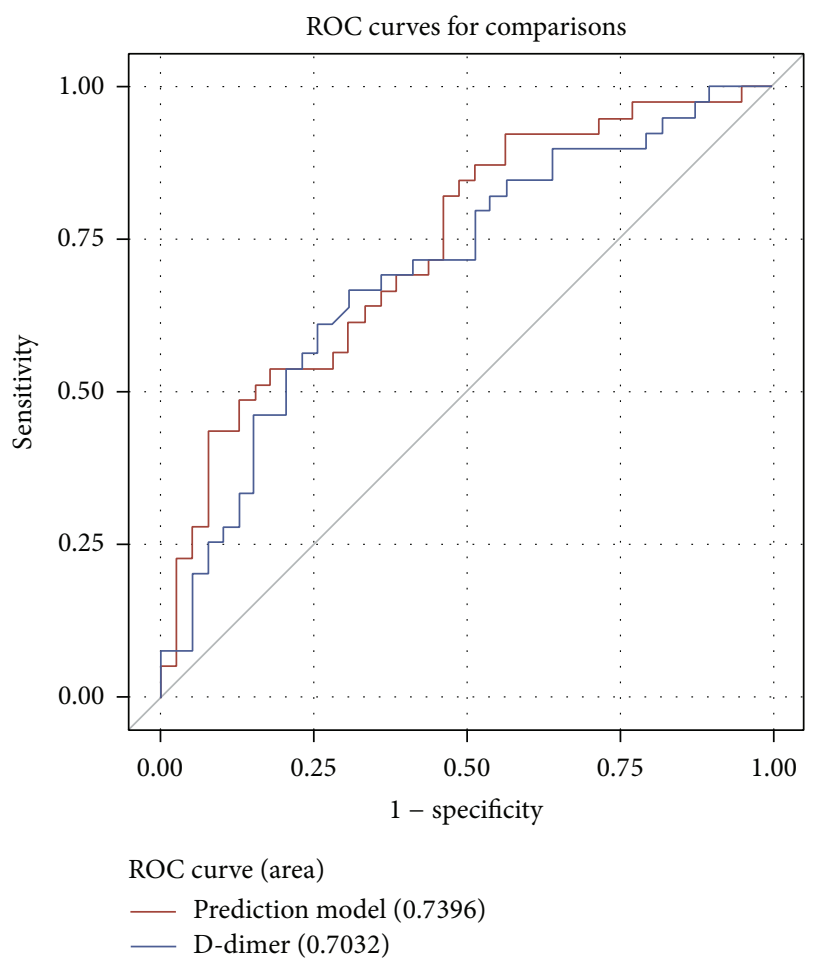

FIgURE 3: ROC curves for D-dimers and prediction model.

scale, that is, as odds ratios. Table 5 presents the studied covariates along with their levels and the coded values we used in the models. Results on AIC from the multiple conditional logistic regression models are presented in Table 6. According to AIC values, illustrated in Table 6, the model with fever (model 5) seems to fit worst the data because it yields larger AIC value (AIC 55.33) than the model including only D-dimer (model 1; AIC 54.15), whereas the model including only "consolidation" (model 4) seems to fit better the data, since it has the smallest AIC value (AIC 51.54). Therefore, model 4 will be the prediction model of an underlying PE in patients with pneumonia.

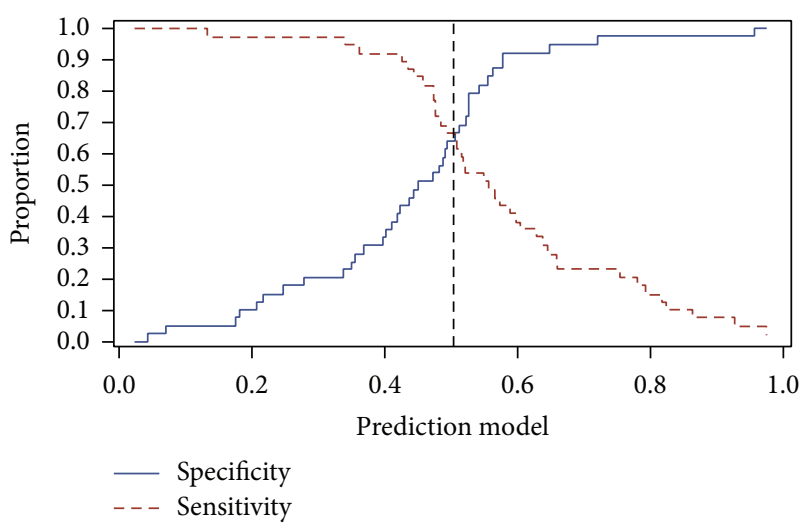

FIGURE 4: Line plot of sensitivity and specificity under all possible cut-offs of the prediction model.

5.6. Comparing the Prediction Model with D-Dimer in Terms of Discriminatory Power. As shown in Figure 3, D-dimer has AUC of 0.703 which reflects low discriminatory power, whereas the prediction model has an AUC of 0.740 . Both parameters reflect quite similar moderate discriminatory power with a small advantage of the prediction model.

Since the prediction model seems to have a little bit better discriminatory power than D-dimer, the last step is to search for the optimal cut-point of the prediction model. Using again the plot by Harris (Figure 4), sensitivity and specificity intersect at the value of 0.50 which corresponds to sensitivity and specificity equal to $64.1 \%$. In order to achieve sensitivity and specificity at least $90 \%$, two cut-points were chosen arbitrarily: $0.30 \mathrm{mg} / \mathrm{L} \mathrm{FEU}$ with sensitivity $97.4 \%$ and specificity $23.1 \%$ and $0.80 \mathrm{mg} / \mathrm{L} \mathrm{FEU}$ with sensitivity $12.8 \%$ and specificity $97.4 \%$. Using the unweighted Youden index, the optimal cut-point is $0.36 \mathrm{mg} / \mathrm{L} \mathrm{FEU}$ with corresponding sensitivity of $92.3 \%$ and specificity of $28.2 \%$.

5.7. Correlation between D-Dimer and Extension of PE and Right Ventricular Hypertension. To answer the questions (i) whether patients with a central embolism are expected to have higher $\mathrm{D}$-dimer than those with subsegmental embolism and (ii) whether patients with a RVH are expected to have higher D-dimer than those without, we implemented a multivariable regression model with $\mathrm{D}$-dimer concentration as dependent variable and the variables embolism ("subsegmental" as reference level) and RVH ("no" as reference level) and their interaction as independent variables.

As shown in Figure 5, in the absence of RVH, D-dimer is on average around $6.24 \mathrm{mg} / \mathrm{L} \mathrm{FEU}$ in patients with central and $4.67 \mathrm{mg} / \mathrm{L} \mathrm{FEU} \mathrm{in} \mathrm{those} \mathrm{with} \mathrm{subsegmental} \mathrm{pulmonary}$ embolism, but in the presence of RVH, D-dimer is on average around $14.45 \mathrm{mg} / \mathrm{L} \mathrm{FEU} \mathrm{in} \mathrm{patients} \mathrm{with} \mathrm{central} \mathrm{as}$ opposed to around $3.38 \mathrm{mg} / \mathrm{L} \mathrm{FEU} \mathrm{in} \mathrm{those} \mathrm{with} \mathrm{subsegmen-}$ tal embolism. Therefore, RVH correlates with D-dimers in patients with central pulmonary embolism but not in those with subsegmental one. 
TABLE 6: Results on AIC from the multiple conditional logistic regression models.

\begin{tabular}{lll}
\hline Model & Included variables & AIC \\
\hline$(1)$ & D-dimer & 54.15 \\
$(2)$ & D-dimer and other symptoms & 55.76 \\
$(3)$ & D-dimer and chest pain & 53.10 \\
$(4)$ & D-dimer and consolidation & 51.54 \\
$(5)$ & D-dimer and fever & 55.33 \\
$(6)$ & D-dimer, other symptoms, and chest pain & 55.69 \\
$(7)$ & D-dimer, other symptoms, and consolidation \\
$(8)$ & D-dimer, chest pain, and consolidation & 53.43 \\
$(9)$ & D-dimer, other symptoms, chest pain, and consolidation & 51.79 \\
\hline
\end{tabular}

AIC: Akaike information criterion.

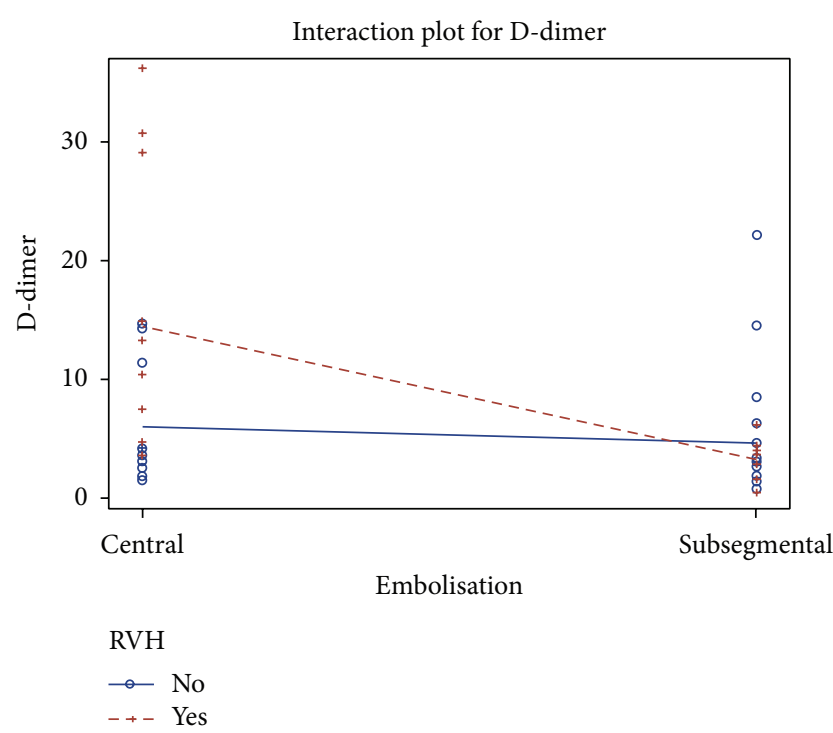

Figure 5: Correlation between D-dimer and extension of PE and right ventricular hypertension.

\section{Discussion}

The diagnosis of pneumonia in emergency setting is based on patient's symptoms, laboratory tests, and chest $\mathrm{X}$-ray consolidation, but the origin remains unclear (infarction pneumonia as a result of pulmonary embolism or community acquired pneumonia caused by infectious pathogens without underlying pulmonary infarction) as long as no CTA is performed. Despite that, not all patients diagnosed with pneumonia can or should undergo a CTA. Therefore, our purpose was to create a model based on parameters that can be easily collected by admission in order to predict which patients with pneumonia are at a high risk for an underlying pulmonary embolism and therefore should undergo a CT angiography of the lung to get the diagnosis confirmed or excluded. We conducted a retrospective study including only subjects where CTA was already performed because this design allows the comparison between the two groups. However, a prospective study could be feasible after having a prediction model as a decision making tool. We collected clinical and paraclinical characteristics on admission and tested them for their diagnostic accuracy separately and in combination.

D-dimer performs moderately but significantly better than CRP and leucocytes count in order to predict an underlying PE in patients with pneumonia. The exclusion of septicemic patients, considering that D-dimer increase in sepsis, brought only a slight increase of the diagnostic accuracy of the marker. The selected threshold of $0.55 \mathrm{mg} / \mathrm{L}$ FEU provides a high sensitivity but a low specificity. This information is already known in the setting of pulmonary embolism [5] but insufficiently investigated in case of concomitant infarction pneumonia [1].

In the question for an optimal D-dimer threshold, we compared a "candidate" cut-off of $2.85 \mathrm{mg} / \mathrm{LFEU}$ where sensitivity and specificity are equal to $68.89 \%$ with the given cut-off at $0.55 \mathrm{mg} / \mathrm{LFEU}$. According to the plot analysis proposed by Harris (REF) sensitivity decreases whereas specificity increases for cut-off value above $2.85 \mathrm{mg} / \mathrm{L} \mathrm{FEU}$ and, in contrast, sensitivity increases whereas specificity decreases for cut-off values below $2.85 \mathrm{mg} / \mathrm{L}$ FEU. There is subsequently no optimal cut-point for $\mathrm{D}$-dimer reaching an acceptable diagnostic ability. Interestingly, an age-adjusted $\mathrm{D}$-dimer value (AADD) has been proposed lately, in order to increase the clinical usefulness of the marker (due to the literature, AADD is calculated as patient's age $* 10 \mathrm{ng} / \mathrm{mL}$, in patients aged $>50$ years) [6]. This calculation was not performable in our protocol, as we initially matched cases and controls according to gender and age.

Considering the clinical symptoms on admission, fever was almost equally present in both categories. This was an interesting finding as most of clinicians combine fever exclusively with infection. Chest pain was clearly more often in the group of PE, but no statistical difference was observed in comparison to the CAP. Shortness of breath (dyspnea) and cough, as long as hemoptysis were observed predominantly in the CAP group, and syncope in the PE group. The distribution of symptoms seems to be different in two similar studies. In the study of Zhang et al. chest pain, shortness of breath, hemoptysis, and fever were identified as independent risk factors of a PE in patients preliminarily diagnosed with pneumonia [7]. On the other hand, Söderberg et al. highlighted presence of dyspnea and/or chest pain in pulmonary embolism and fever, chills, and/or cough in community acquired pneumonia [8]. The reason for the heterogeneous 
symptoms' distribution between similar studies lies more likely in the study design or the symptoms' definition.

Nevertheless, the combination of clinical symptoms with D-dimer in multiple prediction models of an underlying PE did not perform better than D-dimer itself, with the exception of chest pain and X-ray consolidation. Ultimately, we decided to use the prediction model combining X-ray consolidation and D-dimer and compare it with D-dimer itself. ROC curve confirmed a similar discriminating power and plot analysis by Harris retrieved any optional cut-off which could increase the diagnostic accuracy of the prediction model.

In terms of the association between D-dimer, RVH, and extension of pulmonary embolism, we found that RVH correlates with $\mathrm{D}$-dimers in patients with central pulmonary embolism but not in those with subsegmental one. Note that the precision of the results is very low due to a small sample of 45 patients and, hence, any conclusions should be drawn with caution. Moreover, RVH can be observed in many confounding conditions, more often secondary due to COPD or LVI, which practically impoverish the meaning of echocardiographic findings in identifying underlying PE in the setting of pneumonia.

\section{Conclusions}

Our study failed to find a prediction model of an underlying $\mathrm{PE}$ in pneumonia, based on clinical and paraclinical findings, which is significantly better than D-dimer itself. D-dimer is a marker of a moderate ability to identify PE when pneumonia is present. Therefore, we encourage all clinicians to perform a CTA of the lung, once they suspect pulmonary embolism in a patient diagnosed with pneumonia.

\section{Additional Points}

(i) D-dimer has a moderate ability to detect PE in the setting of pneumonia.

(ii) No optimal cut-point for D-dimer seems to reach acceptable diagnostic ability.

(iii) A prediction model, combining D-dimer with clinical symptoms, has a better discriminatory power than Ddimer itself, but not statistically significant.

(iv) By clinical suspicion of a PE in a patient with pneumonia, CTPA should be conducted.

\section{Competing Interests}

None of the authors have any competing interests to disclose.

\section{References}

[1] D. Jiménez Castro, E. Pérez-Rodríguez, L. Montaner, J. Flores, and G. Díaz Nuevo, "Diagnostic value of D dimer in pulmonary embolism and pneumonia," Respiration, vol. 68, no. 4, pp. 371375,2001

[2] K. Scheeres, H. Knoop, M. van der Jos, and G. Bleijenberg, "Clinical assessment of the physical activity pattern of chronic fatigue syndrome patients: a validation of three methods," Health and Quality of Life Outcomes, vol. 7, article 29, 2009.
[3] K. Harris, ROC hard? No, ROC made easy! Proceedings of the 2010 SAS Global Forum, GlaxoSmithKline, UK, http:// support.sas.com/resources/papers/proceedings10/222-2010 .pdf.

[4] N. E. Breslow, N. E. Day, K. T. Halvorsen, R. L. Prentice, and C. Sabai, "Estimation of multiple relative risk functions in matched case-control studies," American Journal of Epidemiology, vol. 108, no. 4, pp. 299-307, 1978.

[5] F. M. Fesmire, M. D. Brown, J. A. Espinosa et al., "Critical issues in the evaluation and management of adult patients presenting to the emergency department with suspected pulmonary embolism," Annals of Emergency Medicine, vol. 57, no. 6, pp. 628-652.e75, 2011.

[6] J. Flores, J. García de Tena, J. Galipienzo et al., "Clinical usefulness and safety of an age-adjusted D-dimer cutoff levels to exclude pulmonary embolism: a retrospective analysis," Internal and Emergency Medicine, vol. 11, no. 1, pp. 69-75, 2016.

[7] Y. Zhang, Q. Zhou, Y. Zou et al., "Risk factors for pulmonary embolism in patients preliminarily diagnosed with communityacquired pneumonia: a prospective cohort study," Journal of Thrombosis and Thrombolysis, vol. 41, no. 4, pp. 619-627, 2016.

[8] M. Söderberg, U. Hedström, M. Sjunnesson, G. Lärfars, and C. Jorup-Rönström, "Initial symptoms in pulmonary embolism differ from those in pneumonia: a retrospective study during seven years," European Journal of Emergency Medicine, vol. 13, no. 4, pp. 225-229, 2006. 


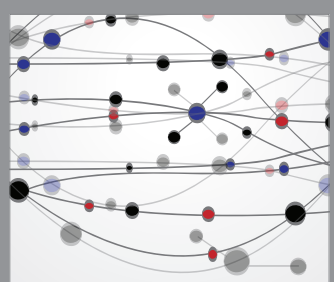

The Scientific World Journal
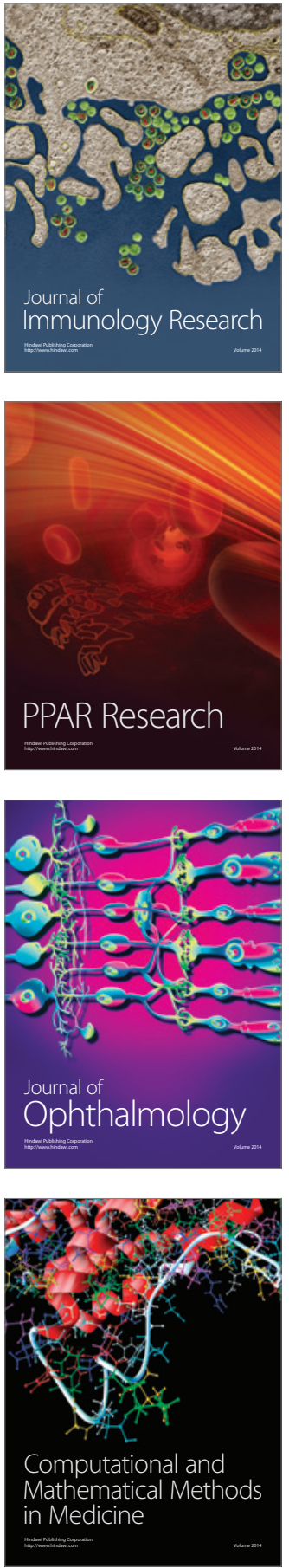



Gastroenterology Research and Practice

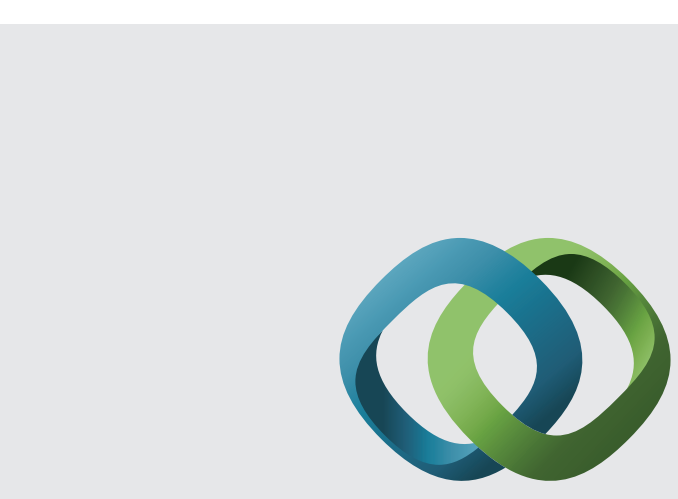

\section{Hindawi}

Submit your manuscripts at

http://www.hindawi.com
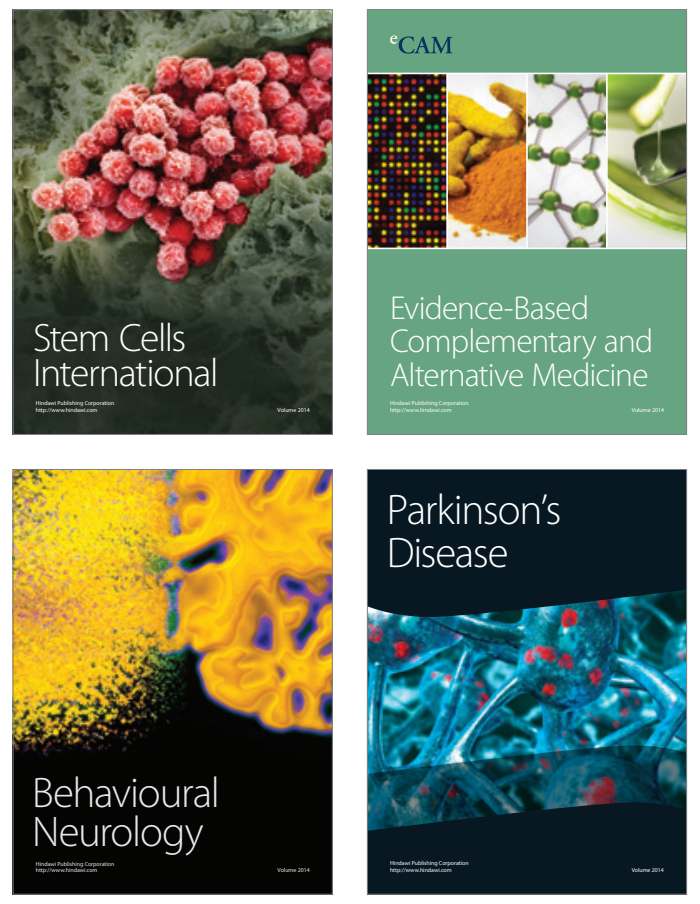
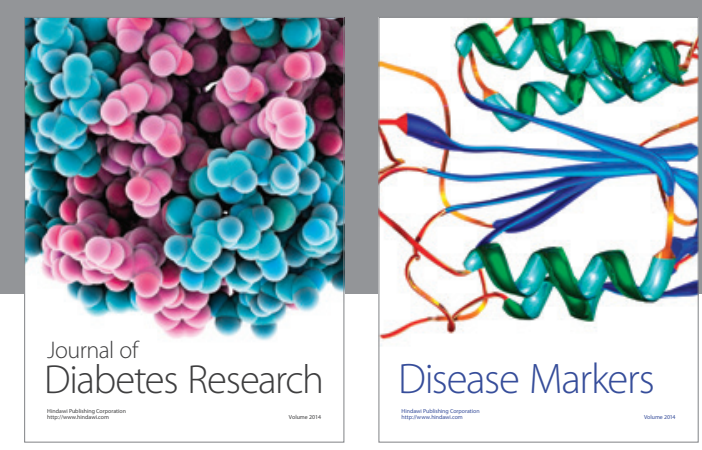

Disease Markers
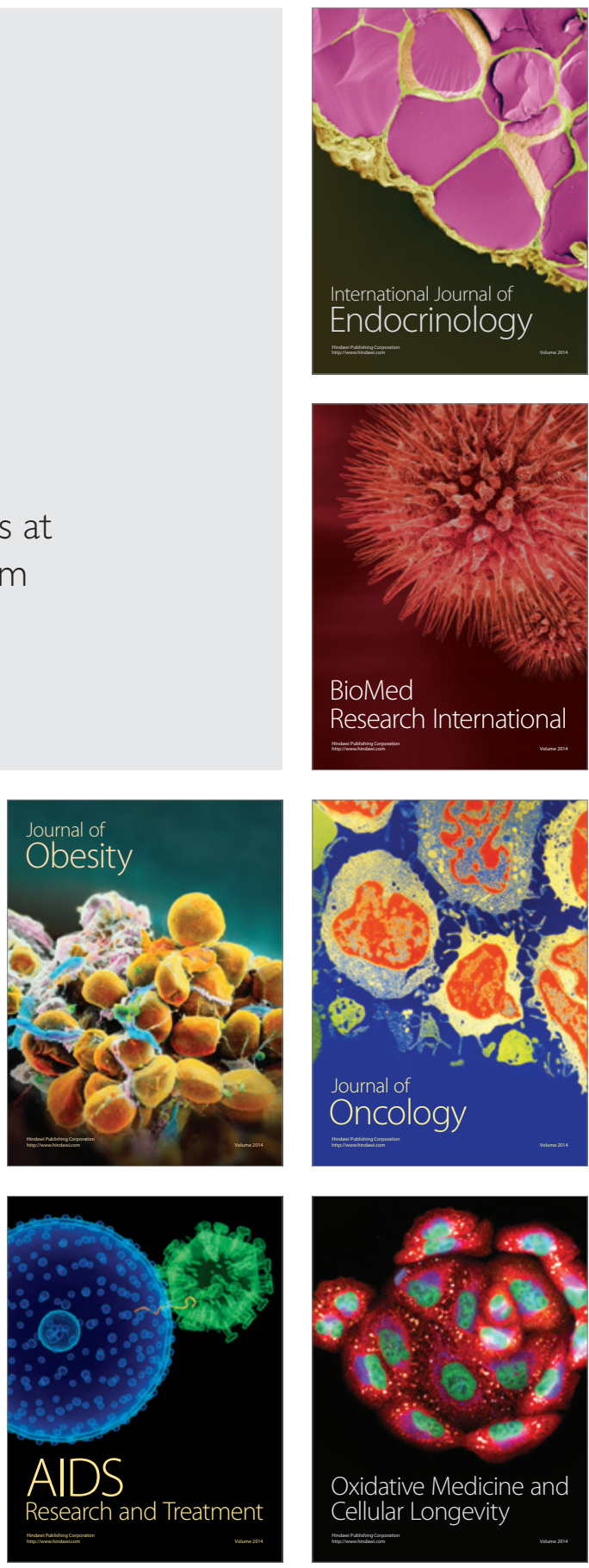